\title{
Cervical cancer screening using the Cervista high- risk human papillomavirus test: opportunistic screening of a hospital-based population in Fujian province, China
}

This article was published in the following Dove Press journal:

Cancer Management and Research

\author{
Guanyu Ruan',* \\ Yiyi Song ${ }^{2, *}$ \\ Binhua Dong' \\ Xiaodan Mao' \\ Fen Lin' \\ Yafang Kang' \\ Shuxia $\mathrm{Xu}^{3}$ \\ Xianjing Chen ${ }^{2}$ \\ Qibin $\mathrm{Wu}^{2}$ \\ Pengming Sun ${ }^{1,2}$ \\ 'Laboratory of Gynecologic Oncology, \\ Fujian Provincial Maternity and \\ Children's Hospital, Affiliated Hospital \\ of Fujian Medical University, Fuzhou, \\ China; ${ }^{2}$ Department of Gynecology, \\ Fujian Provincial Maternity and \\ Children's Hospital, Affiliated Hospital \\ of Fujian Medical University, Fuzhou, \\ China; ${ }^{3}$ Department of Pathology, \\ Fujian Provincial Maternity and \\ Children's Hospital, Affiliated Hospital \\ of Fujian Medical University, Fuzhou, \\ China \\ *These authors have contributed \\ equally to this work
}

Correspondence: Pengming Sun Laboratory of Gynecologic Oncology, Fujian Provincial Maternity and Children's Hospital, Affiliated Hospital of Fujian Medical University, No. 18, Daoshan Road, Gulou District, Fuzhou 35000 I, Fujian, People's Republic of China

Tel +8659 I 87558732

Fax +86 59I 8755 I247

Email sunfemy@hotmail.com

\begin{abstract}
Objectives: The Cervista ${ }^{\circledR}$ high-risk human papillomavirus (HR-HPV) test was evaluated as a primary screening method for cervical cancer in women aged $\geq 21$ years and was compared with different screening and triage combinations.

Materials and methods: A nested case-control study within the Fujian provincial Cervical Lesion Screening Cohorts was used to evaluate the Cervista test as the primary cervical screening method in a hospital-based population. Strategy 1 primarily screened using a cytology screen with HR-HPV testing used for triage. Strategy 2 primarily screened using cytology and HRHPV co-testing. Strategy 3 primarily screened using HR-HPV testing and triaged HPV-positive women based on cytology. Strategy 4 primarily screened using HR-HPV testing and referred A9 pool HPV-positive women to colposcopy directly, whereas non-A9 HPV-positive women were triaged using cytology.
\end{abstract}

Results: There were 10,183 women included in this study; $16.49 \%(1677 / 10,183)$ were HRHPV-positive, 9.52\% had abnormal cytology, and 9907 women were normal during followup. A total of 276 women were diagnosed with cervical intraepithelial neoplasia 2 or worse (CIN2+), 197 with CIN3 or worse (CIN3+), and 70 with cervical cancer. Moreover, $10.15 \%$ (20/197) women who were CIN3+ were identified as cytology-negative, while $8.63 \%(17 / 197)$ were HR-HPV negative $(P>0.05)$. The cumulative risk rate for HPV-/cytology- was 0.836 (95\% CI, 0.424-1.648) in CIN3+ cases. Strategy 4 yielded the highest sensitivity for CIN2+ or $\mathrm{CIN} 3+$ and the lowest positive predictive value for CIN2+ or CIN3+ among the four screening strategies.

Conclusion: The Cervista HR-HPV test can provide a reliable and sensitive clinical reference for the cervical cancer primary screen.

Keywords: human papillomavirus, primary screen, A9 pool, liquid-based cytology, histology

\section{Introduction}

Cervical cancer is thus far the most common human papillomavirus (HPV)-related cancer, ${ }^{1}$ of the 600,000 invasive cancer cases caused by HPV in 2012 , invasive cervical cancer accounted for $>500,000$ of the cases, which resulted in approximately 266,000 deaths. ${ }^{2}$ Persistent infection with high-risk human papillomavirus (HR-HPV) is strongly associated with pre-invasive lower genital tract disease and invasive cancer. ${ }^{3}$ One of the major preventive strategies involves the detection of treatable precancers and early cancers using HPV assays. Cervical cytology screening programs, which are still the most widely used tests, have substantially reduced the incidence of and mortality due to 
cervical cancer, particularly in countries with a wide-screening coverage. ${ }^{4,5}$ However, cytology screening has a lower sensitivity in terms of detecting pre-invasive and invasive cervical lesions than does HPV testing, and the sensitivity of cytology varies according to the laboratory and the expertise of the technologists, as well as the medical infrastructure of a particular region. ${ }^{6,7}$ Moreover, cytology has lower reassurance against prevalent and especially incident pre-cancers and requires shorter intervals between screens to achieve good sensitivity rates. ${ }^{7,8}$ Primary HPV screening has been compared with cytology in several large, randomized clinical trials: HPV testing has been allowed for the earlier detection of precancers and reduced the incidence of cancer during the follow-up. ${ }^{9}$ Accordingly, intervals between screens can be extended. In addition, the reassurance provided by co-testing over HPV testing alone is limited. ${ }^{10}$ Primary HPV screening algorithms have been approved in the US, Netherlands, and Australia and are planned in Italy (2018). ${ }^{11,12}$

China accounts for approximately one-fifth of the world's population, and its cervical cancer burden has a substantial effect on the global estimates of the current and future burden of the disease. ${ }^{13}$ An estimate suggested that the number of new cervical cancer cases in China was 100,000 every year, accounting for $29 \%$ of the world's total. ${ }^{14}$ However, only $21 \%$ of women reported ever undergoing a Papanicolaou (Pap) test in China. The increasing trend in the incidence and mortality due to cervical cancer may be related to inadequate screening, increasing prevalence of HPV infection, and the lack of an HPV vaccine. ${ }^{15}$ Since the HPV vaccine was only recently introduced in China, it will take some time until it has been implemented across China; ${ }^{16}$ therefore, screening alone serves as the major prevention strategy during this period. In 2009, China's government launched the National Cervical Cancer Screening Program in rural areas. It was the first time that the Chinese government had proposed to gradually widen access to cervical cancer screening services in rural areas, and it represented a step toward the nationwide provision of cervical cancer screening. ${ }^{14}$ Although the coverage of cervical cancer screening in China is low, ${ }^{17,18} \mathrm{a}$ more sensitive primary HPV testing method was required in clinical practice.

The US Food and Drug Administration (FDA) has approved four kinds of HPV tests: Hybrid Capture ${ }^{\circledR} 2$ (HC2) HPV DNA (Qiagen, Hilden, Germany), Cervista ${ }^{\circledR}$ (Hologic, Massachusetts, USA), APTIMA ${ }^{\circledR}$ (Hologic, Massachusetts, USA), and the cobas ${ }^{\circledR}$ HPV test (Roche Diagnostics, Indianapolis, USA). Thus far, the FDA has recommended the cobas HPV test alone for HPV testing for primary screening. This led to speculation that primary cervical cancer screening via HPV testing is a promising screening strategy that needs further validation, especially in Chinese women. A negative $\mathrm{HC} 2,{ }^{19} \mathrm{GP} 5+/ 6+$ polymerase chain reaction-enzyme immunoassay (PCR-EIA), ${ }^{19,20}$ or Cobas HPV test ${ }^{21,22}$ indicates a low risk of developing cervical precancer or cancer in follow-up data of HPV-based primary screen. The Cervista HPV HR test was the second HR-HPV assay approved by the FDA in 2009 and is a qualitative test that uses three separate oligonucleotide mixtures (A5/A6 pool [HPV 51, 56, and 66], A7 pool [18, 39, 45, 59, and 68], and A9 pool [HPV 16, 31, $33,35,52$, and 58]) depending on the correlation among 14 types of HR-HPV DNA gene sequences used to detect HRHPV types. ${ }^{23}$ The Cervista test detects 14 HR-HPV types: HPV66 and the same 13 HR-HPV types as detected by $\mathrm{HC} 2$ testing, and several comparative studies have shown its similar sensitivity and specificity to those of the HC2 test. ${ }^{23}$ However, the utility of the Cervista HPV test as a primary HPV screening method and the clinical performance of different cervical cancer screening strategies, particularly in the Chinese population, remains unclear. This study was designed to provide evidence of the efficiency of a cervical cancer screening strategy using the Cervista HPV test for the detection of cervical intraepithelial neoplasia grade 2 or more severe $(\mathrm{CIN} 2+)$ and $\mathrm{CIN} 3$ or more severe $(\mathrm{CIN} 3+)$ in a Chinese hospital-based population.

\section{Materials and methods Study population}

We conducted a nested case-control study within the Fujian provincial Cervical Lesion Screening Cohorts (FCLSCs), a cervical screening cohort study involving a hospital-based and community-based population in Fujian Provincial Maternity and Children's Health Hospital. This study is an opportunistic screening of a hospital-based population. Women, who underwent primary cervical screening, including the Cervista HR-HPV test, were initially included from 2012 to 2016. The population eligible for this cohort study involved two arms, one consisting of healthy patients undergoing routine physical examinations, and another consisting of patients visiting the outpatient clinic for any gynecologic conditions except cancer. All cases assigned to this nested case-control study which fulfilled the following criteria were included as cases or controls: 1) sexually active nonpregnant women aged $\geq 21$ years; 2 ) valid Cervista HPV testing and cytology results; 3) consistently tested with Cervista or cytology in the follow-up phase; and 4) willingness to participate in this study. Cases included women with a confirmed diagnosis of 


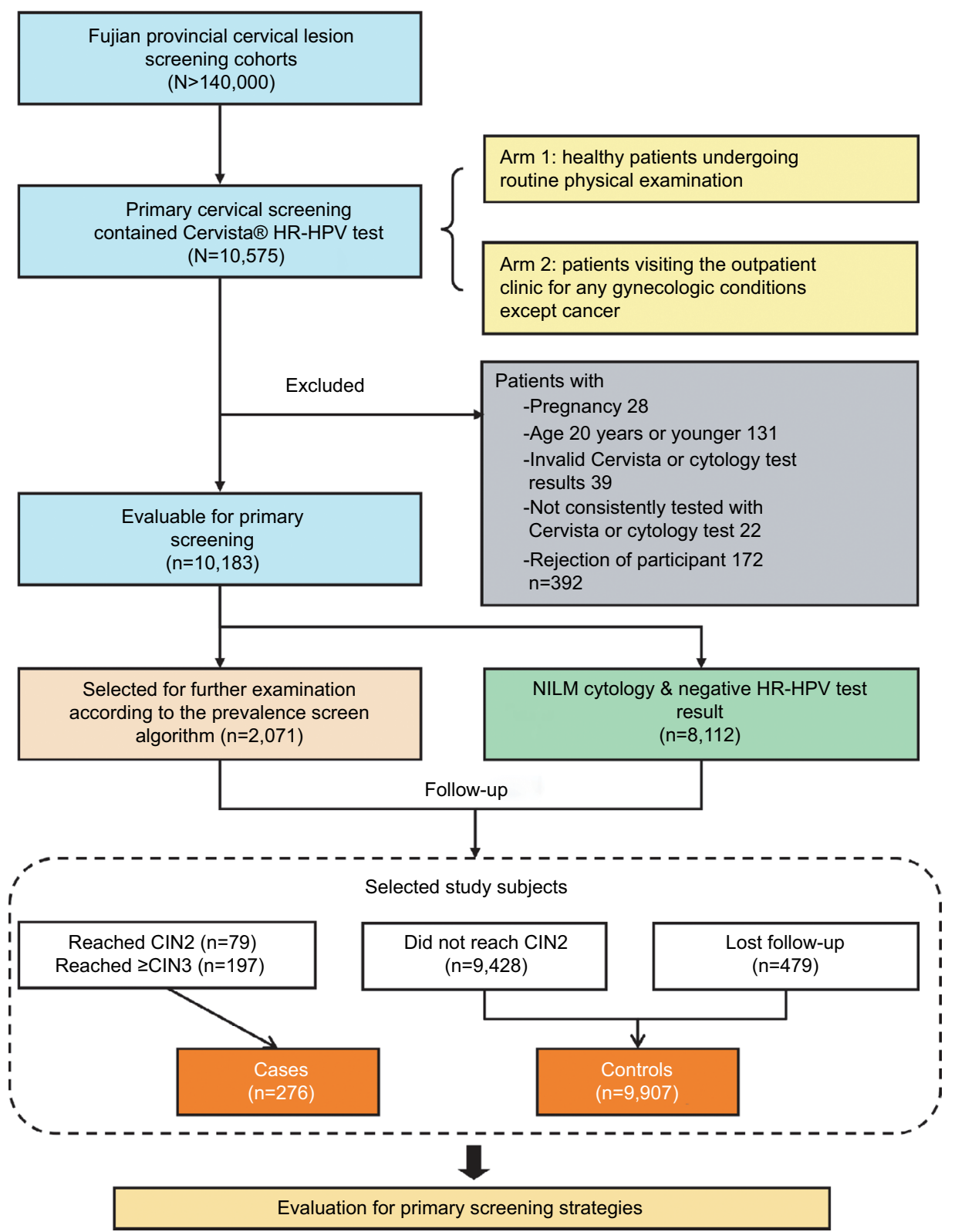

Figure I Flowchart of the screening profiles of women in the nested case-control study.

Abbreviations: HR-HPV, high-risk human papillomavirus; NILM, negative for intraepithelial lesion and malignancy; CIN, cervical intraepithelial neoplasia.

CIN2 or worse. Controls included women with none of the above diagnoses during the follow-up. All individuals in this study provided written informed consent. The study protocol was approved by the Hospital Ethics Committee of Fujian Provincial Maternity and Children's Hospital, Affiliated Hospital of Fujian Medical University. The study flowchart is shown in Figure 1.

\section{Cervical specimen collection}

Cervical cells were collected using plastic brushes from the cervical canal of all participants and placed into $20-\mathrm{mL}$ vials of PreservCyt ${ }^{\circledR}$ solution (Hologic, USA) for cytology or HPV DNA testing. The samples for cytology and HPV assays were stored at $4^{\circ} \mathrm{C}$ and then sent to laboratory for testing.

\section{Liquid-based cytology}

ThinPrep slides were blinded and evaluated, independent from the results of the other assays, by two experienced cytopathologists. If the diagnoses were different, the cervical samples were reviewed again, and a consensus diagnosis was obtained. The results were evaluated using the 2001 Bethesda system. Samples were classified as negative for intraepithelial 
lesion and malignancy (NILM), atypical squamous cells of undetermined significance (ASC-US), low-grade squamous intraepithelial lesion (LSIL), atypical squamous cells, not possible to exclude high-grade squamous intraepithelial lesion (ASC-H), high-grade squamous intraepithelial lesion (HSIL), squamous cervical cancer (SCC), atypical glandular cells (AGC), and adenocarcinoma in situ (AIS). ${ }^{24}$

\section{Cervista HR-HPV assay}

The Cervista HR-HPV assay used magnetic beads for DNA purification and Invader $^{\circledR}$ chemistry for signal amplification, to qualitatively detect the specific nucleic acid sequences of 14 HR-HPV types simultaneously in three different pools (A5/A6 pool: 51, 56, and 66; A7 pool: 18, 39, 45, 59, and 68; A9 pool: $16,31,33,35,52$, and 58). ${ }^{25}$ All detection procedures and interpretation of the HPV results were conducted in accordance with the manufacturer's instructions. ${ }^{26}$ The human histone 2 gene (HIST2H2BE) served as an internal control for cellular DNA present in the sample. A positive result indicated that at least one of high-risk types is present in the groups.

\section{Colposcopy and histology}

Women who were HPV-positive and/or had an abnormal cytological result (with a grade higher than ASC-US) were referred for colposcopy and punch biopsy within 12 weeks of the initial visit. Women with a punch biopsy diagnosis greater than HSIL underwent a loop electrosurgical excision procedure cone biopsy or conization using a cold knife. Specimens were fixed in $10 \%$ formalin and routinely processed for paraffin embedding. Subsequently, 4- $\mu$ m-thick histological sections were cut and stained with hematoxylin and eosin using the standard method. Cervical biopsy specimens were then histologically examined and classified according to the CIN system. ${ }^{27}$ The primary round was included from the first visit to complete colposcopy with biopsy or endocervical curettage (ECC) if necessary. All women who underwent colposcopy in the primary round and who did not have CIN2+ were eligible for the follow-up round. For women with negative results for cytology and HPV tests at primary round, as the disease status, it was assumed that no new disease would be observed until further histology was received.

\section{Screening procedures}

This study evaluated the performance of four screening strategies using the dataset created by the study. A flowchart of the strategies conducted is shown in Figure 2. The three strategies currently used for cervical cancer screening (primary cytology test, co-testing, and primary HR-HPV test) and another strategy were designed to evaluate the performance of the Cervista HR-HPV test. Strategy 1 comprised cytology with HPV testing performed only for ASC-US. Strategy 2 comprised co-testing women with both cytology and HRHPV testing, after which HPV-positive women with negative cytology were retested with both tests in a routine follow-up per year and underwent ECC if either test yielded abnormal results. Strategy 3 comprised primary screening of women with HPV testing alone, triage of HPV-positive women with cytology, and referring those with cytology $\geq$ ASC-US/AGC for colposcopy/ECC. Strategy 4 comprised screening women using HPV testing, referring those infected with A9 pool for colposcopy/ECC, triage for those without infection with A9 pool with cytology, and referring those with cytology $\geq$ ASC-US/AGC for colposcopy/ECC.

\section{Statistical analysis}

The performance characteristics of the screening strategies were evaluated by calculating the sensitivity, specificity, positive predictive value (PPV), negative predictive value (NPV), positive likelihood rate (PLR), negative likelihood rate (NLR), and Youden index according to the standard definitions for CIN2+ or CIN3+. The 95\% CIs were calculated using exact binomial CIs. The number of colposcopies needed to detect one case was equivalent to the number colposcopies per case, identified by end point. The $\chi^{2}$ test, Fisher's exact test, and cumulative risk analysis were performed. Results for women with missing disease status in primary or followup round were imputed that the characteristics of women with valid biopsy results are not significantly different from those with missing disease status. All data analyses were performed using SPSS 17.0 (SPSS Inc., Chicago, IL, USA). All statistical tests were two-sided, and a $P$-value $<0.05$ was considered to indicate statistical significance.

\section{Results}

\section{Clinical characteristics of the studied population}

A total of 10,575 cases of primary cervical screening used the Cervista HR-HPV test in FCLSCs; of these, 10,183 cases fulfilled the criteria. The median follow-up time was 2.5 years ( $1-5$ years). During the course of follow-up, 2071 women were selected for further examination according to the prevalence screen algorithm; 1592 had available cervical histology results. A flow chart describing the selection of the study cohort with inclusion/exclusion criteria can be found in Figure 1. The total HR-HPV positivity rate using 

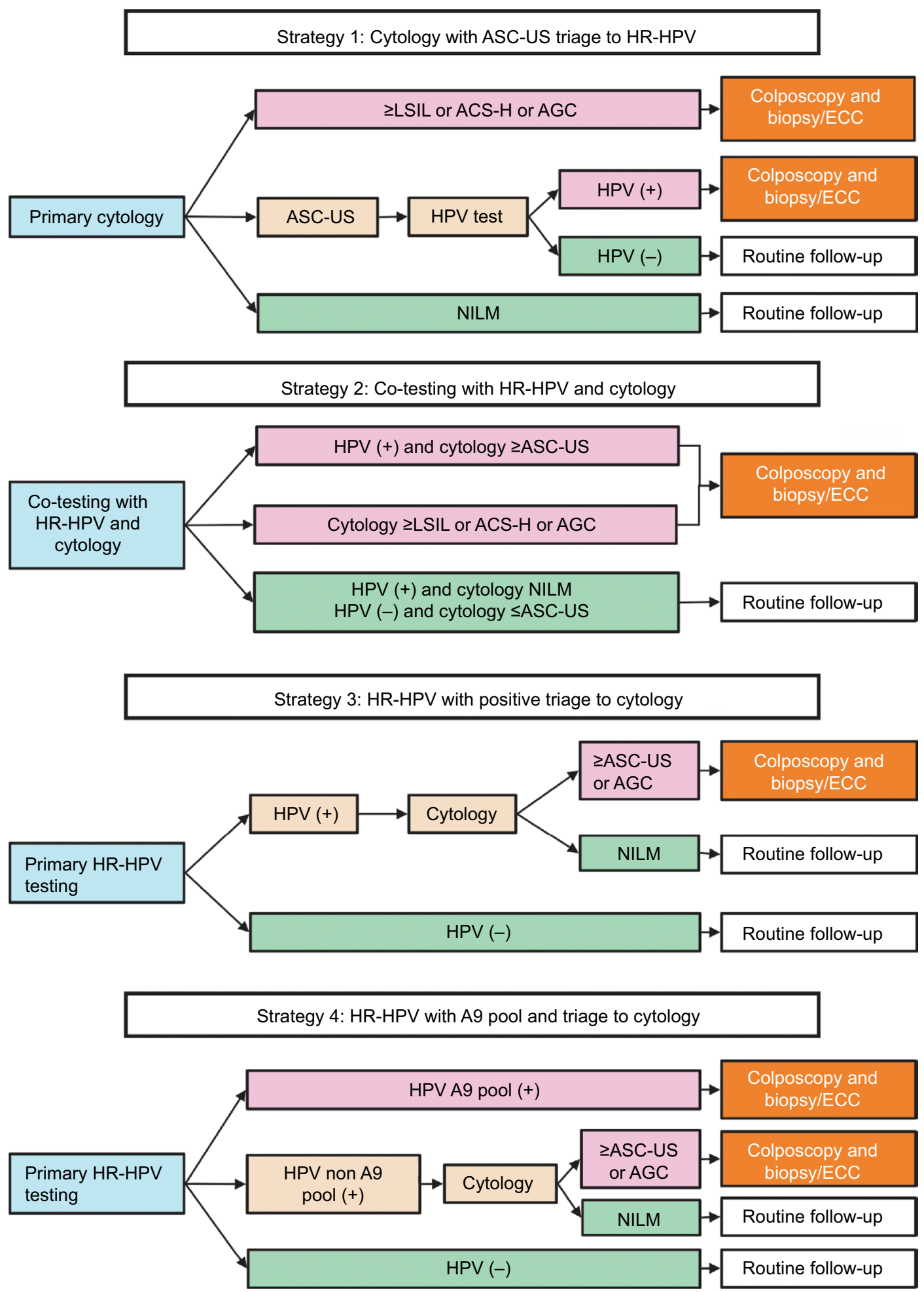

Figure 2 Flowchart of the four screening strategies for cervical cancer.

Abbreviations: ASC-US, atypical squamous cells of undetermined significance; HR-HPV, high-risk human papillomavirus; LSIL, low-grade squamous intraepithelial lesion; ASC-H, atypical squamous cells, not possible to exclude high-grade squamous intraepithelial lesion; AGC, atypical glandular cells; ECC, endocervical curettage; NILM, negative for intraepithelial lesion and malignancy; HPV, human papillomavirus. 
the Cervista HR test was $16.49 \%$. The frequencies of A5/ A6, A7, and A9 pools were 4.44\%, 4.45\%, and $10.30 \%$, respectively. Moreover, $9.52 \%$ of women had abnormal cytology: ASC-US, 4.21\%; AGC, 1.10\%; LSIL, 1.91\%; ASC-H, $0.19 \%$, HSIL, $0.92 \%$, and cervical cancer, $0.56 \%$. Among women who had available histology results, a total of 276 were diagnosed with CIN2+, 197 with CIN3+, and 70 with cervical carcinoma (Table 1).

\section{Cumulative risk for $\mathrm{CIN} 2+, \mathrm{CIN} 3+$, or cancer}

Herein, 20 of 197 (10.15\%) cases of CIN3+ identified during the study occurred in women with negative baseline cytology. In contrast, 17 (8.63\%) occurred in women who tested negative for HR-HPV during the study $(P>0.05)$. The cumulative risk rate was 0.836 (HPV-/cytology-, 95\% CI: 0.424-1.648) in CIN3+ cases. Similar results were observed using a CIN2+ end point, and the cumulative risk rate was 0.701 (HPV-/cytology-, 95\% CI: 0.403-1.221). Moreover, 68 of $70(97.14 \%)$ women with cervical cancers were HPVpositive, and 66 of $70(94.28 \%)$ had $\geq$ ASC-US cytology. The cumulative risk rate was 0.485 (95\% CI: $0.086-2.740)$ for HPV negative and cytology negative results.

\section{Identification of cervical disease among the four screening strategies}

Of the four screening strategies that were evaluated, strategy 4 had the highest sensitivity (89.85\%; 95\% CI: 85.63-94.07) for the detection of CIN3+ (Table 2). In comparison, the sensitivities of strategy 1 and strategy 2 were $88.32 \%$ (95\% CI: 83.84-92.81) and that of strategy 3 (HR-HPV primary) was $81.73 \%$ (95\% CI: 76.33-87.12). Strategy 3 had the highest specificity for CIN3+ (95.84\%; 95\% CI: 95.45-96.24), and strategy 4 had the lowest specificity (89.55\%; 95\% CI: 88.95-90.15). Strategy 1 and strategy 2 had an intermediate specificity that was between those of the other two strategies. The Youden's index for strategy 1 and 2 for detecting CIN3+ was higher than that of the other strategies $(82.99 \%$ vs. $77.57 \%$ or $79.39 \%)$. Similar results were observed using CIN2+ as the end point. The PPV and NPV, as well as PLR and NLR are shown in Table 2. In our study, strategy 1 had a similar sensitivity, specificity, PPV, NPV, PLR, and NLR as that of strategy 2 to detect CIN2+ or $\mathrm{CIN} 3+$, but strategy 1 required fewer tests than strategy 2 to detect CIN2+ or CIN3+.

Strategy 4 detected a higher number of CIN3+ cases in the primary round than any of the other three strategies (Table 3). For example, strategy 4 detected $9.94 \%$ more cases of $\mathrm{CIN} 3+$ in the primary round than strategy 3 . However, strategy 4 resulted in an increase in the number of colposcopies in the primary round and an increase in the number of colposcopies per case of CIN3+ in the primary round (5.42). Comparable results were seen using a CIN2+ end point.

\section{Discussion}

Cervical cancer is the second most common gynecological malignancy in women in developing countries, where approximately $85 \%$ of cervical cancer cases culminate in death; it is preventable because of its long precancerous lesion status. ${ }^{6}$ However, limited sensitivity and a correspondingly limited NPV, limits the use of cytology testing, although cytology testing has greatly reduced the incidence and the mortality of cervical cancer in developed countries when implemented as a primary screening test. Persistent HR-HPV infection plays an important role in the development of cervical cancer. Therefore, research on the value of the HPV-based screening has been performed in many institutions. ${ }^{7}$ The HC2, ${ }^{19}$ GP5+/6+ PCR-EIA, ${ }^{19,20}$ and Cobas HPV tests ${ }^{21,22}$ are considered fully clinically and epidemiologically viable HPV assays, validated following the Meijer validation protocol. ${ }^{28}$ The Cervista HPV HR test was the second HR-HPV assay

Table I Results of the HR-HPV testing and cytology for cervical disease

\begin{tabular}{llll}
\hline & Total & CIN2+ & CIN3+ \\
\hline Number & 10,183 & 276 & 197 \\
HR-HPV (+) & 1677 & 252 & 180 \\
\% of number; 95\% Cl & $16.49 ; 15.75-17.20$ & $91.30 ; 87.34-94.35$ & $91.37 ; 86.54-94.89$ \\
Cytology $\geq$ ASC-US & 970 & 243 & 177 \\
\% of number; 95\% Cl & $9.52 ; 8.96-10.11$ & $88.04 ; 83.62-91.63$ & $89.85 ; 84.76-93.69$ \\
\hline
\end{tabular}

Note: ${ }^{\text {a }}$ The $\mathrm{Cl}$ calculation was based on the exact $\mathrm{Cl}$ for that proportion.

Abbreviations: CIN, cervical intraepithelial neoplasia; HR-HPV, high-risk human papillomavirus; ASC-US, atypical squamous cells of undetermined significance. 
Table 2 Detection of cervical disease using different screening strategies for HR-HPV

\begin{tabular}{|c|c|c|c|c|c|c|c|}
\hline $\begin{array}{l}\text { Screening } \\
\text { Strategies }\end{array}$ & $\begin{array}{l}\text { Sensitivity } \\
(\%, 95 \% \mathrm{Cl})\end{array}$ & $\begin{array}{l}\text { Specificity } \\
(\%, 95 \% \mathrm{CI})\end{array}$ & $\begin{array}{l}\text { PPV } \\
(\%, 95 \% \mathrm{Cl})\end{array}$ & $\begin{array}{l}\text { NPV } \\
(\%, 95 \% \mathrm{CI})\end{array}$ & $\begin{array}{l}\text { PLR } \\
(\%, 95 \% \mathrm{CI})\end{array}$ & $\begin{array}{l}\text { NLR } \\
(\%, 95 \% \mathrm{CI})\end{array}$ & YI (\%) \\
\hline \multicolumn{8}{|c|}{ CIN2+ endpoint } \\
\hline I & $\begin{array}{l}86.59 \\
(82.57-90.61)\end{array}$ & $\begin{array}{l}95.28 \\
(94.86-95.69)\end{array}$ & $\begin{array}{l}33.80 \\
(30.32-37.29)\end{array}$ & $\begin{array}{l}99.61 \\
(99.48-99.74)\end{array}$ & $\begin{array}{l}18.33 \\
(16.59-20.26)\end{array}$ & $\begin{array}{l}0.14 \\
(0.10-0.19)\end{array}$ & 81.87 \\
\hline 2 & $\begin{array}{l}86.59 \\
(82.57-90.61)\end{array}$ & $\begin{array}{l}95.28 \\
(94.86-95.69)\end{array}$ & $\begin{array}{l}33.80 \\
(30.32-37.29)\end{array}$ & $\begin{array}{l}99.61 \\
(99.48-99.74)\end{array}$ & $\begin{array}{l}18.33 \\
(16.59-20.26)\end{array}$ & $\begin{array}{l}0.14 \\
(0.10-0.19)\end{array}$ & 81.87 \\
\hline 3 & $\begin{array}{l}80.80 \\
(76.15-85.44)\end{array}$ & $\begin{array}{l}96.44 \\
(96.07-96.80)\end{array}$ & $\begin{array}{l}38.72 \\
(34.74-42.69)\end{array}$ & $\begin{array}{l}99.45 \\
(99.30-99.60)\end{array}$ & $\begin{array}{l}22.68 \\
(20.16-25.50)\end{array}$ & $\begin{array}{l}0.2 \\
(0.16-0.25)\end{array}$ & 77.23 \\
\hline 4 & $\begin{array}{l}89.49 \\
(85.88-93.11)\end{array}$ & $\begin{array}{l}90.17 \\
(89.58-90.75)\end{array}$ & $\begin{array}{l}20.23 \\
(17.98-22.48)\end{array}$ & $\begin{array}{l}99.68 \\
(99.56-99.79)\end{array}$ & $\begin{array}{l}9.1 \\
(8.47-9.78)\end{array}$ & $\begin{array}{l}0.12 \\
(0.08-0.16)\end{array}$ & 79.66 \\
\hline CIN3+ end & & & & & & & \\
\hline I & $\begin{array}{l}88.32 \\
(83.84-92.81)\end{array}$ & $\begin{array}{l}94.66 \\
(94.22-95.10)\end{array}$ & $\begin{array}{l}24.61 \\
(21.44-27.79)\end{array}$ & $\begin{array}{l}99.76 \\
(99.66-99.86)\end{array}$ & $\begin{array}{l}16.55 \\
(15.02-18.23)\end{array}$ & $\begin{array}{l}0.12 \\
(0.08-0.18)\end{array}$ & 82.99 \\
\hline 2 & $\begin{array}{l}88.32 \\
(83.84-92.81)\end{array}$ & $\begin{array}{l}94.66 \\
(94.22-95.10)\end{array}$ & $\begin{array}{l}24.61 \\
(21.44-27.79)\end{array}$ & $\begin{array}{l}99.76 \\
(99.66-99.86)\end{array}$ & $\begin{array}{l}16.55 \\
(15.02-18.23)\end{array}$ & $\begin{array}{l}0.12 \\
(0.08-0.18)\end{array}$ & 82.99 \\
\hline 3 & $\begin{array}{l}81.73 \\
(76.33-87.12)\end{array}$ & $\begin{array}{l}95.84 \\
(95.45-96.24)\end{array}$ & $\begin{array}{l}27.95 \\
(24.29-31.62)\end{array}$ & $\begin{array}{l}99.63 \\
(99.5-99.75)\end{array}$ & $\begin{array}{l}19.67 \\
(17.53-22.06)\end{array}$ & $\begin{array}{l}0.19 \\
(0.14-0.26)\end{array}$ & 77.57 \\
\hline 4 & $\begin{array}{l}89.85 \\
(85.63-94.07)\end{array}$ & $\begin{array}{l}89.55 \\
(88.95-90.15)\end{array}$ & $\begin{array}{l}14.5 \\
(12.52-16.47)\end{array}$ & $\begin{array}{l}99.78 \\
(99.68-99.87)\end{array}$ & $\begin{array}{l}8.59 \\
(7.98-9.26)\end{array}$ & $\begin{array}{l}0.11 \\
(0.07-0.17)\end{array}$ & 79.39 \\
\hline
\end{tabular}

Abbreviations: HR-HPV, high-risk humanpapillomavirus; PPV, positive predictive value; NPV, negative predictive value; PLR, positive likelihood rate; NLR, negative likelihood rate; YI, Youden index; CIN, cervical intraepithelial neoplasia.

Table 3 Detection of cervical disease using different screening strategies and the number of coloposcopies that each strategy requires

\begin{tabular}{|c|c|c|c|c|c|c|}
\hline $\begin{array}{l}\text { Screening } \\
\text { strategies }\end{array}$ & Total & $\begin{array}{l}\text { No. of } \\
\text { detected cases } \\
\text { at primary } \\
\text { round }\end{array}$ & $\begin{array}{l}\text { No. of } \\
\text { detected cases } \\
\text { at follow-up } \\
\text { round }\end{array}$ & $\begin{array}{l}\text { No. lost to } \\
\text { follow-up } \\
\text { at primary } \\
\text { round }\end{array}$ & $\begin{array}{l}\text { No. of } \\
\text { colposcopies } \\
\text { at primary } \\
\text { round }\end{array}$ & $\begin{array}{l}\text { No. of } \\
\text { colposcopies to } \\
\text { detect one case at } \\
\text { primary round }\end{array}$ \\
\hline \multicolumn{7}{|c|}{ CIN2+ endpoint } \\
\hline 1 & 276 & 239 & 37 & 52 & 655 & 2.74 \\
\hline 2 & 276 & 239 & 37 & 52 & 655 & 2.74 \\
\hline 3 & 276 & 223 & 53 & 51 & 525 & 2.35 \\
\hline 4 & 276 & 247 & 29 & 262 & 959 & 3.88 \\
\hline \multicolumn{7}{|c|}{ CIN3+ endpoint } \\
\hline I & 197 & 174 & 23 & 52 & 655 & 3.76 \\
\hline 2 & 197 & 174 & 23 & 52 & 655 & 3.76 \\
\hline 3 & 197 & 161 & 36 & 51 & 525 & 3.26 \\
\hline 4 & 197 & 177 & 20 & 262 & 959 & 5.42 \\
\hline
\end{tabular}

Abbreviation: CIN, cervical intraepithelial neoplasia.

approved by the FDA in 2009 and introduced into China by the China Food and Drug Administration (CFDA) in 2011, with a similar sensitivity and specificity to those of the $\mathrm{HC} 2$ test. ${ }^{23}$ However, the clinical performance of Cervista as a primary HPV screening method, especially in a Chinese hospital-based population, has remained uncertain.

FCLSCs are cervical screening cohorts involving a hospital-based and community-based population since 2008 in Fujian Provincial Maternity and Children's Health Hospital, a local cervical screen center. The clinical performance characteristics of PCR-reverse dot blot (PCR-RDB) assays among 10,442 women in FCLSCs were evaluated previously; the results suggested that PCR-RDB can provide a reliable and sensitive clinical reference for cervical cancer screening. ${ }^{29}$ In the current study, patient data were derived from that for FCLSCs using the Cervista test since 2012; moreover, the population eligible for this cohort study involved two arms, one consisting of healthy patients undergoing routine physical examinations, and another consisting of patients visiting the outpatient clinic for any gynecologic conditions except cancer. The total infection rate of HR-HPV tested using the Cervista HR-HPV test was estimated at $16.49 \%$, which is in 
accordance to our previous report ${ }^{29}$ and other epidemiological studies regarding HPV in China. ${ }^{30}$ We also found that $10.15 \%$ women with CIN3+ were cytology-negative. In contrast, $8.63 \%$ women with CIN3+ were HR-HPV negative $(P>0.05)$. The cumulative risk rate was 0.836 (HPV-/ cytology-, 95\% CI: 0.424-1.648) in CIN3+ cases, implying that this hospital-based population was representative for evaluating the strategy regarding cervical cancer screening.

Currently, there are three main strategies for HPV testing: as a triage for ASC-US cytology, testing all women with both HPV and cervical cytology (co-testing) results, and primary HPV testing with cytology or colposcopy triage. ${ }^{31-33}$ In our study, these three main strategies were used to evaluate the Cervista test. We found that the sensitivity of strategy 1 (cytology) and strategy 2 (co-testing) was $88.32 \%$ (95\% CI: 83.84-92.81) and that of the strategy 3 (HR-HPV primary) was $81.73 \%$ (95\% CI: 76.33-87.12). The results were similar to those of a previous study. ${ }^{23}$ Therefore, these data suggest that Cervista HR-HPV testing was robust for HPV screening in Chinese women. ${ }^{22}$

The most obvious triage approaches are cytology and HPV16/18 genotyping, both of which have been discussed widely in the literature. ${ }^{7}$ In our previous study, HPV-16 was the most prevalent genotype in the HR-HPV-positive women, followed by, from highest to lowest, HPV-52, $-58,-18,-53$, $-33,-51,-56,-59,-68,-31,-66,-39,-35$, and $-45 .{ }^{29}$ The data implied that the A9 pool (HPV-16, -31, -33, -35, -52, and -58) involved approximately $77 \%$ HPV positive genotypes and were the major HPV group in CIN2+ cases, compared to the A7 pool (HPV-18, $-39,-45$, and -59 ) at $14 \%$ and the A5/ A6 pool (HPV-51, -56, and -66) at 6\%. Based on the A9 pool, which is a major species in Fujian, we also evaluated HR-HPV primary screening for A9 pool as a triage approach to explore the Cervista test role in a primary screen. Strategy 4 (HR-HPV primary screening for A9 pool) had the highest sensitivity at $89.85 \%$ for detecting CIN3+. These results are similar to those of the ATHENA study, wherein the HPV primary screening had the highest sensitivity and identified more CIN3+ compared to that of the cytology or hybrid strategy. Furthermore, strategy 4 required the most colposcopies to detect one case of high-grade CIN. Moreover, strategies 1, 2, and 3 had fewer losses to follow-up at the primary round, in which the lifetime risk of cervical cancer was reduced by $25 \%-35 \% .{ }^{34}$ In general, the Cervista HR-HPV test can provide a reliable and sensitive clinical reference for a cervical cancer primary screen.

An estimate suggests that the number of new cervical cancer cases in China accounted for $29 \%$ of the new cases of cervical cancer in the world. ${ }^{14}$ In China, a hospital-based cervical screen is currently the main strategy for cervical cancer prevention. Nevertheless, the coverage of cervical cancer screening in China is still much lower than that in developed countries. ${ }^{17,18}$ Therefore, a more sensitive primary HPV testing method is required in clinical practice. Since 2011 when Cervista as approved by the CFDA, many hospitals initiated use of the technology in clinical practice and explored how applications of the Cervista test would be meaningful and effective in Chinese women.

Based on the above analysis, strategy 4, which used the Cervista HPV HR test as an initial screening method and referred those infected with A9 pool for colposcopy, triage for those without infection with A9 pool, and referring those with cytology $\geq$ ASC-US for colposcopy could be a suitable option for cervical cancer screening in Chinese women.

\section{Acknowledgments}

The authors would like to acknowledge the contribution of Tingting Lin and Lili Chen for kindly helping to collect data and Meimei Huang and Weiyi Huang for advice in preparation of figures. This work was supported by the Fujian Provincial Key Projects in Science and Technology (grant no. 2015YZ0002-1) and by Fujian Provincial Healthy Innovation Project (grant no. 2009-CXB-33).

\section{Disclosure}

The authors report no conflicts of interest in this work.

\section{References}

1. Plummer M, de Martel C, Vignat J, Ferlay J, Bray F, Franceschi S. Global burden of cancers attributable to infections in 2012: a synthetic analysis. Lancet Glob Health. 2016:4(9):e609-e616.

2. Globocan. Cervical Cancer estimated incidence, mortality and prevalence worldwide in 2012. Available from: http://globocan.iarc.fr/Pages/ fact_sheets_cancer.aspx. Accessed July 20, 2017.

3. de Sanjosé S, Diaz M, Castellsagué X, Clifford G, Bruni L, Muñoz N, Bosch FX. Worldwide prevalence and genotype distribution of cervical human papillomavirus DNA in women with normal cytology: a metaanalysis. Lancet Infect Dis. 2007;7(7):453-459.

4. Vaccarella S, Franceschi S, Engholm G, Lonnberg S, Khan S, Bray F. 50 years of screening in the Nordic countries: quantifying the effects on cervical cancer incidence. Br J Cancer. 2014;111(5):965-969.

5. Vaccarella S, Lortet-Tieulent J, Plummer M, Franceschi S, Bray F. Worldwide trends in cervical cancer incidence: impact of screening against changes in disease risk factors. Eur J Cancer. 2013;49(15):3262-3273.

6. Goodman A. HPV testing as a screen for cervical cancer. $B M J$. 2015:350:h2372.

7. Arbyn M, Ronco G, Anttila A, et al. Evidence regarding human papillomavirus testing in secondary prevention of cervical cancer. Vaccine. 2012;30(Suppl 5):F88-F99.

8. Schiffman M, Doorbar J, Wentzensen N, et al. Carcinogenic human papillomavirus infection. Nat Rev Dis Primers. 2016;2:16086.

9. Ronco G, Dillner J, Elfström K, et al. Efficacy of HPV-based screening for prevention of invasive cervical cancer: follow-up of four European randomised controlled trials. Lancet. 2014;383(9916): 524-532. 
10. Gage JC, Schiffman M, Katki HA, et al. Reassurance against future risk of precancer and cancer conferred by a negative human papillomavirus test. J Natl Cancer Inst. 2014;106(8):dju153.

11. Schiffman M, Doorbar J, Wentzensen N, et al. Carcinogenic human papillomavirus infection. Nat Rev Dis Primers. 2016;2:16086.

12. Huh W, Ault K, Chelmow D, et al. Use of primary high-risk human papillomavirus testing for cervical cancer screening: interim clinical guidance. Gynecol Oncol. 2015;136(2):178-182.

13. Di J, Rutherford S, Chu C. Review of the cervical cancer burden and population-based cervical cancer screening in China. Asian Pac J Cancer Prev. 2015;16(17):7401-7407.

14. Women's health in rural China. Lancet. 2009;374(9687):358.

15. Wang R, Guo XL, Wisman GB, et al. Nationwide prevalence of human papillomavirus infection and viral genotype distribution in 37 cities in China. BMC Infect Dis. 2015;15:257.

16. Jiang X, Tang H, Chen T. Epidemiology of gynecologic cancers in China. J Gynecol Oncol. 2018;29(1):e7.

17. Wang B, He M, Chao A, et al. Cervical cancer screening among adult women in China, 2010. Oncologist. 2015;20(6):627-634.

18. Zhao F, Hu SY, Zhang SW, Chen WQ, Qiao YL. Cervical cancer mortality in 2004 -2005 and changes during last 30 years in China. Zhonghua Yu Fang Yi Xue Za Zhi. 2010;44(5):408-412.

19. Dillner J, Rebolj M, Birembaut P, et al. Long term predictive values of cytology and human papillomavirus testing in cervical cancer screening: joint European cohort study. BMJ. 2008;337:a1754.

20. Elfström KM, Smelov V, Johansson AL, et al. Long term duration of protective effect for HPV negative women: follow-up of primary HPV screening randomised controlled trial. BMJ. 2014;348:g130.

21. Arbyn M, Snijders PJ, Meijer CJ, et al. Which high-risk HPV assays fulfil criteria for use in primary cervical cancer screening? Clin Microbiol Infect. 2015;21(9): 817-826.

22. Wright TC, Stoler MH, Behrens CM, Sharma A, Zhang G, Wright TL. Primary cervical cancer screening with human papillomavirus: end of study results from the ATHENA study using HPV as the first-line screening test. Gynecol Oncol. 2015;136(2):189-197.

23. Boers A, Wang R, Slagter-Menkema L, et al. Clinical validation of the Cervista HPV HR test according to the international guidelines for human papillomavirus test requirements for cervical cancer screening. J Clin Microbiol. 2014;52(12):4391-4393.
24. Solomon D, Davey D, Kurman R, et al. The 2001 Bethesda System: terminology for reporting results of cervical cytology. JAMA. 2002;287(16):2114-2119.

25. Day SP, Hudson A, Mast A, et al. Analytical performance of the Investigational Use Only Cervista HPV HR test as determined by a multi-center study. J Clin Virol. 2009;45 Suppl 1:S63-S72.

26. Cervista ${ }^{\mathrm{TM}}$ HPV HR.Available from: https://www.hologic.com/sites/ default/files/package-insert/15-3100_105_01.pdf. Accessed July 20, 2017.

27. Waxman AG, Chelmow D, Darragh TM, Lawson H, Moscicki AB. Revised terminology for cervical histopathology and its implications for management of high-grade squamous intraepithelial lesions of the cervix. Obstet Gynecol. 2012;120(6):1465-1471.

28. Meijer C, Berkhof J, Castle PE, et al. Guidelines for human papillomavirus DNA test requirements for primary cervical cancer screening in women of 30 years and older. Int J Cancer. 2009;124(3):516-520.

29. Sun P, Song Y, Ruan G, et al. Clinical validation of the PCR-reverse dot blot human papillomavirus genotyping test in cervical lesions from Chinese women in the Fujian province: a hospital-based population study. J Gynecol Oncol. 2017;28(5):e50.

30. Pan QJ, Hu SY, Guo HQ, et al. Liquid-based cytology and human papillomavirus testing: a pooled analysis using the data from 13 populationbased cervical cancer screening studies from China. Gynecol Oncol. 2014;133(2):172-179.

31. ASCUS-LSIL Traige Study (ALTS) Group. Results of a randomized trial on the management of cytology interpretations of atypical squamous cells of undetermined significance. Am J Obstet Gynecol. 2003;188(6):1383-1392.

32. Saslow D, Solomon D, Lawson HW, et al. American Cancer Society, American Society for Colposcopy and Cervical Pathology, and American Society for Clinical Pathology screening guidelines for the prevention and early detection of cervical cancer. J Low Genit Tract Dis. 2012;16(3):175-204.

33. Huh WK, Ault KA, Chelmow D, et al. Use of primary high-risk human papillomavirus testing for cervical cancer screening: interim clinical guidance. Gynecol Oncol. 2015;136(2):178-182.

34. Goldie SJ, Gaffikin L, Goldhaber-Fiebert JD, et al. Cost-effectiveness of cervical-cancer screening in five developing countries. $N$ Engl J Med. 2005;353(20):2158-2168.
Cancer Management and Research

\section{Publish your work in this journal}

Cancer Management and Research is an international, peer-reviewed open access journal focusing on cancer research and the optimal use of preventative and integrated treatment interventions to achieve improved outcomes, enhanced survival and quality of life for the cancer patient. The manuscript management system is completely online and includes

\section{Dovepress}

a very quick and fair peer-review system, which is all easy to use. Visit http://www.dovepress.com/testimonials.php to read real quotes from published authors. 